\title{
SmartParking: A Secure and Intelligent Parking System Using NOTICE
}

\author{
Gongjun Yan, Stephan Olariu, Michele C. Weigle, Mahmoud Abuelela
}

\begin{abstract}
Parking is costly and limited in almost every major city in the world. Innovative parking systems for meeting near-term parking demand are needed. In this paper, we propose a novel, secure and intelligent parking system based on the concept and framework of NOTICE [WO07], a secure and privacy-aware architecture for the notification of traffic incidents. The proposed system, called SmartParking, is a serviceoriented intelligent parking system through which drivers can view and reserve a parking spot on the fly. The parking process can then be a straightforward and non-stop process. More importantly, SmartParking is a secure and privacy-aware parking system. The proposed infrastructure prevents most security/privacy attacks. We address hardware/software architecture and implementations. The evaluation of this proposed system proves its efficiency.
\end{abstract}

\section{INTRODUCTION}

Parking is limited in almost every major city in the world contributing to traffic congestion, air pollution, and driver frustration. For example, the Manhattan Central Business District (CBD) has 109,222 off-street public parking spots [NYC06], for a ratio of approximately one off-street public spot for every $16 \mathrm{CBD}$ workers. Yet, often parking spots are wasted. In large parking lots, a driver may exit the lot without knowing about new spots that have just become vacant. Finding an empty parking spot may also lead to driver frustration if another car takes the spot before the driver can reach it.

Thus, innovative parking systems for meeting near-term parking demand are needed. With wireless communications, computer, control, and electronics technologies, intelligent service-oriented parking management can improve parking space utilization and improve driver experience. In this paper, we propose a novel, secure and intelligent parking application system based on the concept and framework of NOTICE [WO07], [AOW08], which is a secure and privacyaware architecture for the notification of traffic incidents. The proposed system called SmartParking is a security/privacy aware parking system. Parking information, order information, and vehicle information are protected by the NOTICE infrastructure. The proposed infrastructure prevents most security/privacy attacks. The proposed SmartParking is an intelligent parking system. Drivers can view and reserve

This work is supported by U.S.A NSF (CNS 0721586)

G. Yan, Computer Science Department, Old Dominion University, Norfolk, VA 23529, USA ygong june CS. odu . edu

S. Olariu, Computer Science Department, Old Dominion University, Norfolk, VA 23529, USA olariuecs.odu. edu

M. C. Weigle, Computer Science Department, Old Dominion University, Norfolk, VA 23529, USA mweigle@ cs . odu. edu

M. Abuelela, Computer Science Department, Old Dominion University, Norfolk, VA 23529, USA eabuecs.odu. edu a parking spot on the fly. The parking process can be a straightforward and non-stop process.

\section{A. Related Work}

Caliskan et al. [CGM06] proposes a parking system in which parking automats are the producers of resource reports. The infrastructure uses IEEE 802.11 to broadcast these reports as raw text packets. The report packet size is 92 bytes. The assumption is that parking automats are able to sense their occupation status at any moment. Each vehicle starts with an empty cache, i.e. it has not obtained any resource report. During its trip, it receives resource reports from parking automats or other vehicles. Received reports are integrated into a vehicle's cache. These reports are aggregated and disseminated among vehicles. The decision strategy of which parking lot is used is based on two influencing parameters: the age of a resource and the distance to a resource. This system is not concerned with security or privacy issues, which are important.

The basic infrastructure of a parking system relies on devices to detect if parking spaces are occupied. Several approaches are employed to detect reliable information. First wired sensors are widely used [MK00]: 1) inductive loops, 2) pneumatic road tubes, 3) magnetic sensors, 4) piezoelectric sensors, 5) weigh-in-motion systems. Wolff et al. [WHG $\left.{ }^{+} 06\right]$ use the Earth's magnetic field to detect parking spaces. These devices or sensors are physically wired to the control computers. One shortcoming of the wired sensor systems is that long and complicated wiring is required from parking lots to the central control unit. Also, the cost for developing this system is high because a large amount of sensor units are required. Therefore some wireless sensors can be applied to the parking space detection. Tang et al. [TZC06] developed such a system using Crossbow Mote products and the extended Crossbow XMesh network architecture. Benson et al. propose RF transceiver and antenna with an ATMega 128L micro-controller system $\left[\mathrm{BOO}^{+} 06\right]$. Third, image processing is applied to detect the vehicles [TYI04], [ZZL07]. Funck et al. uses images to detect the parking space [FMO04]. However these methods may incorrectly detect parking vehicles. One example is that a vehicle temporally uses one slot to park in another slot, or that a vehicle just happens to be in the intersection of these sensors. Panayappan et al. [PTSP07] propose a parking system in VANET to locate the available parking lots and spots. This system uses roadside units to relay parking messages and GPS to locate vehicle position. Roadside units maintain the security certificates and parking information. The security issue addressed in this paper is to ensure fare-play among drivers by encryption and frequently 
sensing the available spots. The greedy drivers are prevented from gaining more advantage from the system by lying. But there are some security problems. For example, a roadside attacker pretending to be a vehicle can reserve as many slots as he wishes. In our system, all the communications are triggered by physical pressure on belts and are enabled by short range signals. There is no way to launch a roadside attack.

\section{B. Our Contribution}

The proposed parking system is a novel infrastructure which addresses both security/privacy issues and efficient services. Our main contributions are:

- Privacy is considered in our infrastructure. Vehicles' privacy information is transmitted from belt to belt or belt to parking site, instead of being exposed to other vehicles.

- Security of transaction and user information is considered. The communication is activated only when a vehicle's front wheels press on a belt. This mechanism prevents most security attacks. For example, roadside malicious hackers cannot eavesdrop on the communication.

- Parking sites can frequently publish their advertisements. The advertisement can be transmitted through wireless transceivers and updated on the NOTICE belt infrastructure.

- Drivers can view and reserve parking spots on the fly. A non-stop parking service can be provided to drivers.

\section{NOTICE}

The underlying philosophy of NOTICE [WO07], [AOW08] is that the decision about traffic-related information dissemination should rest with the infrastructure and not with individual vehicles that may have incorrect or incomplete information about the road.

The infrastructure in NOTICE is obtained by embedding sensor belts in the road at regular intervals (e.g., every mile or so), as illustrated in Figure 1. Each belt consists of a collection of piezoelectric pressure sensors, a simple aggregation and fusion engine, and a few small transceivers. The pressure sensors in each belt allow every message to be associated with a physical vehicle passing over the belt, eliminating the need for vehicles to be uniquely identified while avoiding the security problems outlined in the VANET literature [HvL04], $\left[\mathrm{ABD}^{+} 06\right]$. There are two immediate benefits of using belts over roadside infrastructure. First, the belts are far less prone to tampering and, second, they are better placed to detect passing cars and to interact with them in a simple and secure fashion.

Vehicles in NOTICE are fitted with a Tamper-Resistant Device (TRD), for example, Event Data Recorder (EDR) [YOW08], [HvL04], much like the well-known black-boxes on-board aircraft. All of the vehicle's sub-assemblies, including the wireless transceiver unit, speedometer, gas tank

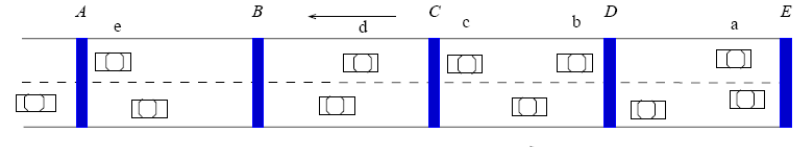

Fig. 1. A collection of belts on a two-lane road. Belts are labeled with capital letters, and cars are labeled with lowercase letters. The figure is not drawn to scale as belts are placed at least $1 \mathrm{~km}$ apart.

reading, tire pressure sensors, and sensors for outside temperature, feed their own readings into the TRD. As a consequence, given a time interval $I$ of interest, the TRD can store information such as the highest and lowest speed during $I$, the position and time of the strongest deceleration during $I$, as well as location $p$, time $t$ and target lane in a lane change.

\section{A. Belt to belt communications}

Each belt is fitted with a few transceivers and the belts do not communicate with each other directly. Instead, adjacent belts rely on passing vehicles to communicate. Referring to Figure 1 featuring a two-lane roadway, where each lane on the roadway has its own dedicated belt. For example, belt $C$ consists of two logical sub-belts, each serving the lanes in one direction. In the case of a divided highway, belts on opposite sides of the median are connected by direct wired connection under the median.

If belt $C$ has a message $m$ to the next belt, $B$, it will encrypt $m$. To pass the encrypted message $m$ to belt $B$, belt $C$ will upload $m$ onto passing car $d$ (as will be described below). When car $d$ reaches belt $B$, the message $m$ will be dropped off and decoded by belt $B$. In turn, belt $B$ may decide to send a message to belt $A$. This would be done using the symmetric key $\mu(B, A, t)$, known only to belts $B$ and $A$.

\section{B. Belt to car communications}

Referring to Figure 1, once the pressure sensors in belt $C$ have detected the front wheels of car $c$, a radio transceiver in the belt will send, at a very low power a "Hello" beacon on a standard control channel containing the ID, $C$, of the belt, as well as handshaking information. If there is trafficrelated information that concerns car $c$, belt $C$ will upload this information to the car. Belt $C$ may also upload a message $m$ destined for the next belt, $B$. Message $m$ is encrypted. The communication time between a belt and a car has been proven to be sufficient [AOW08].

\section{Car to car communications}

Referring again to Figure 1, assume that belt $D$ has an emergency message to convey to belt $C$. Belt $D$ having encrypted the message will upload the message unto car $b$ and will also set the "urgent" bit indicating that car $b$ must try to contact cars traveling in the direction toward $C$ forwarding the encrypted message by radio. 


\section{ARCHITECTURE}

\section{A. Hardware Architecture}

Each vehicle is deployed with a short range wireless transceiver and a simple processor. The transmission range is $1 \mathrm{~m}$. It can be one of the current short range devices, such as ZigBee devices, Bluetooth devices and infrared devices. Both wireless transceiver and processor are fitted into the TRD.

The infrastructure for a parking provider consists of a wireless transceiver, parking belts, InFrared Devices (IFD) and a control computer. The wireless transceiver can be part of a Wireless LAN (WLan) network. It is used to transmit the parking information (for example, the capacity of empty parking spots, the position list of empty parking spots) and reservation information. The parking belts and IFD, as shown in Figure 5, will work together to check-in a vehicle. The IFD at the parking spot connects to a signal light. A yellow light means empty spot. A blue light means filled spot. A red light means misfilled vehicles which will be charged a fine. In some scenarios, vehicles may use a spot to back up or pass through. To prevent misdetection of vehicles, we use both IFD and belts to double check if the vehicles are behaving correctly.

\section{B. Software Architecture}

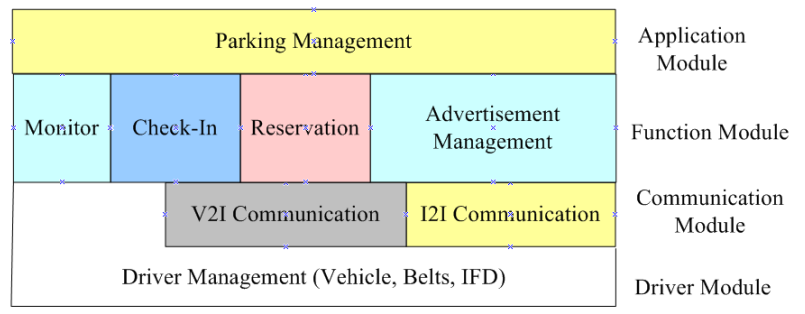

Fig. 2. SmartParking Software Architecture

In the software architecture, we propose several modules shown in Figure 2. The architecture consists of four modules:

- Driver Module is responsible for the communication with hardware devices. The driver module consists of the sensor driver for belts, communication driver for the vehicle's short range transceiver, and IFD driver for vehicle detection.

- Communication Module receives and transmits messages between a sender and a receiver. For Vehicle-toInfrastructure (V2I) communication, this module simplifies the communication process and performs error control, for example verifying the checksum and correcting errors. In general, the purpose is to improve communication speed and enhance message correctness because the communication response time is strict. For Infrastructure-to-Infrastructure (I2I) communication, this module exchanges message between two fixed transceivers, for example between parking lot transceiver and booth transceiver.
- Function Module is the core function of the parking system, including monitor, check-in, reservation, advertisement management. Thanks to the subscription to the driver module and the communication module, the function module can talk to hardware devices and transmit/receive data without having to know the details of the lower levels.

- Application Module manages the whole parking system. The main function of the application includes account management (cash, credit/debit management), operation management, fault tolerance and maintenance management.

\section{SySTEM OpERATIONS}

\section{A. Advertisement Publishing}

A computer center at the parking lot booth frequently updates parking capacity through a map of empty spots in the parking area. This spot information is obtained by connected IFD and parking belts. All information is encrypted or encoded during the communication between the parking transceiver and the road side transceiver to enhance data security. This prevents some attacks, for example eavesdropping messages.

Advertisement of the parking spot information is published by the wireless communication network. The infrastructure for the parking spot advertisement consists of several wireless transceivers. The topology and the number of the transceivers depends on the geography of the parking garage. In Figure 3, we deploy several transceivers along the road and one at the parking site. The transceiver at the parking site is called the base station because it is the center of the transaction. The ones on the roadside connect to belts. They can communicate with each other. The transceiver and the belt shown as $S_{i}$ and $B_{i}$ in Figure 3, which first meet the oncoming traffic from right to left is called publisher station. The advertisements sent by the base station are picked up by vehicles from this station. The transceiver and the belt shown as $S_{i+1}$ and $B_{i+1}$ in Figure 3, which meet the oncoming traffic after publisher station, is called order station. The spot reservation orders are collected from vehicles and transmitted to the base station from this station. There are three order stations $S_{i+1}-B_{i+1}, S_{i+2}-B_{i+2}$ and $S_{i+3}-B_{i+3}$ in Figure 3. The confirmation of order will be sent to the last orders station $S_{i+3}-B_{i+3}$ and carried back to the other order station where the order maker can pick it up.

The process of advertisement publishing is the following: the computer center frequently feeds the up-to-date parking capacity information to the base station. The base station then transmits the parking information to the publisher station. Vehicles pass this belt at publisher station and receive a copy of the parking advertisement by communication with the belt. The advertisement includes the parking site location, the capacity of the empty spots, and the layout of empty spots. 


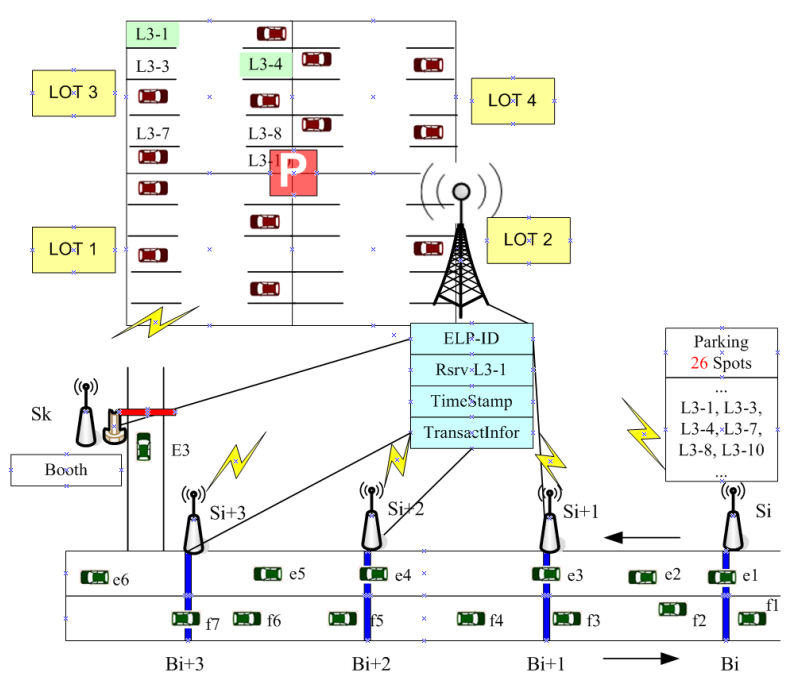

Fig. 3. SmartParking Layout

\section{B. Reserve Parking Spot}

User terminals (e.g. PDAs, cell phones, vehicle display, and laptops) can read the output of the TRD. The received advertisement information is stored in the TRD to prevent modification of the parking information by attackers. Maps of parking sites can be preloaded/downloaded into the user terminals. The format of the software interface is the Extensible Markup Language (XML). These terminals can display the empty spots in a user-friendly manner. An example interface of the parking spots is shown in Figure 4.

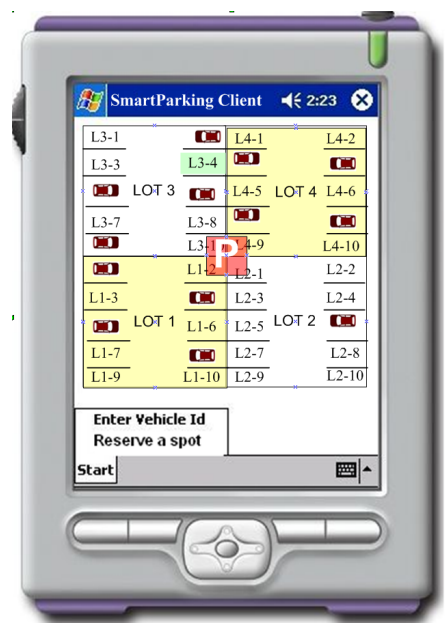

Fig. 4. SmartParking User Interface. A user can view the empty spots and reserve one spot. The reserved spot in this figure is at lot 3 number 4 spot: L3-4

The process of a reservation order will be described using Figure 3. A vehicle $e_{3}$ passes the blet $B_{i}$ at publisher station $\left(S_{i}-B_{i}\right)$. It picks up an advertisement. If $e_{3}$ selects one of the parking spots, for instance $L 3-4$, the user terminal/client program will send a reservation request and make an reservation order which includes the vehicle's Electronic License Plate
ID(ELP-ID) and the selected parking spot number. Suppose $e_{3}$ selects the spot before it passes belt $B_{i+1}$. When $e_{3}$ passes $B_{i+1}$, the transceiver will drop the reservation order to belt $B_{i+1}$. The belt will generate a timestamp and transaction expire time information. These information will be appended to the reservation order message and form a new complete reservation order. The new reservation order is sent to the base station by order station $S_{i+1}-B_{i+1}$ and processed by computer center. The feedback of this order is sent back to the last order station $S_{i+3}-B_{i+3}$. The vehicles in opposite traffic of $e_{3}$ will mule the encrypted feedback message to the order station $S_{i+2}-B_{i+2}$ by picking and dropping this message on belts. Therefore $e_{3}$ can get this feedback message either on belt $B_{i+2}$ or $B_{i+3}$. Since the belts are $1 \mathrm{~km}$ apart each other, the travel time from one belt to another belt is about $1000(\mathrm{~m}) / 31(\mathrm{~m} / \mathrm{s})=32($ seconds $)$ if the average speed of vehicles is 70 miles/hour (i.e. about $31 \mathrm{~m} / \mathrm{s}$ ). This travel time is good enough to cover the reservation order processing time, transmission delay, etc.

Each reservation order consists of a vehicle's ELP-ID, the reserved parking spot number, a timestamp, and transaction expiration time information. The transaction expiration time is the maximum time to drive from the belt where the order is generated to the parking site. If the vehicle orders a spot but does not show up before the transaction expiration time, the reserved spot is freed and the vehicle has to reserve another parking spot.

If a vehicle does not reserve any parking spots and drives directly to the parking lot, the parking entry booth will randomly pick an empty spot for the vehicle. If several reservations collide, new randomly selected parking spots are picked from the empty spots to solve the collision. If the collided spot is the last spot, the spot will be given to the vehicle with the earlier timestamp. Other vehicles will be informed that the spot is not available anymore.

\section{Cancel Transaction}

If a driver decides to cancel a parking reservation, the cancelation process is similar to the reservation process. A cancel order is dropped to the order belt and transmitted to the base station where the cancel order is processed. Feedback is sent back to the last order station and is carried by opposite traffic until the order maker picks it up at an order station belt.

In some special scenarios, a driver might not cancel the transaction. For example, the driver changes mind to continue drive instead of parking and he forgets to cancel the reserved slot. The slot reserved by this driver will be freed when the transaction expiration time is past. To prevent this scenario, a fine can be charged because this driver has held parking resources for a period of time.

\section{Parking Check In}

When vehicle $e_{3}$ with a valid order arrives at the parking entry booth $S_{k}$, the vehicle transceiver can communicate with the booth transceiver and report the encrypted reservation order to the booth transceiver. The reservation order is 
transmitted to the control center where the transaction is validated, a map guidance to the parking spot is calculated. The control center then transmits the confirmation and the map guidance to the boothes where these information is printed and served to the driver.

Vehicle $e_{3}$ uses the map to find the parking spot. Several devices, as shown in Figure 5, are used to detect and validate the vehicle. A top overview of a parking lot is shown in Figure 6. An infrared device can detect vehicle $e_{3}$ taking the spot and set a count-down waiting time (e.g. $1.5 \mathrm{~min}$ ) for the vehicle to fully finish parking. The Belt- $a$ is embedded inside the parking slots. The communication initiates when front wheels of the parking vehicle press on Belt-a. The vehicle transceiver in the TRD reports the encrypted parking confirmation to the transceiver in Belt-a. Belt-a then reports it to the computer center who validates the parked vehicle. The validation result is signaled by lights. A blue light means valid. A red light means misfilled. The Belt- $a$ and the infrared device connect to each other to double check vehicle misparking. When the count-down waiting time reaches to zero, the belts Belt- $a$ and infrared device are activated. Again, the vehicle's transceiver reports the confirmation message to the belt Belt- $a$ who will relay this message to computer center to check. The reason we use both belt and infrared device is that other vehicles may happen to temporarily use a slot to move into a slot or back a vehicle. If the driver is confirmed as misparking, the driver will be charged a fine. As long as a vehicle is parking at a slot, a message will be reported to the control center that the spot is taken. The report consists of parking start timestamp, parking type (valid parking or invalid parking), and confirmation message. All Belt-as can communicate with the computer center by wired or wireless connections.

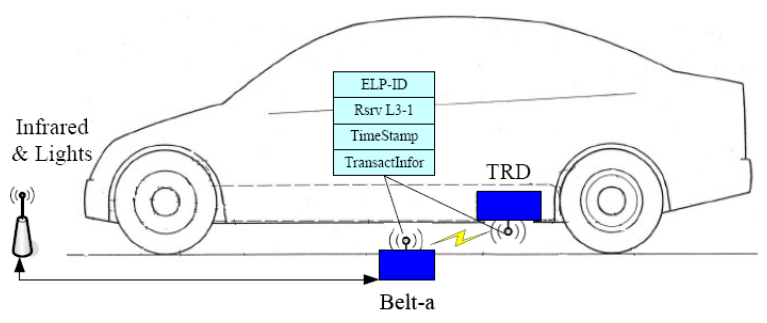

Fig. 5. Vehicle Detection and Transaction Confirm

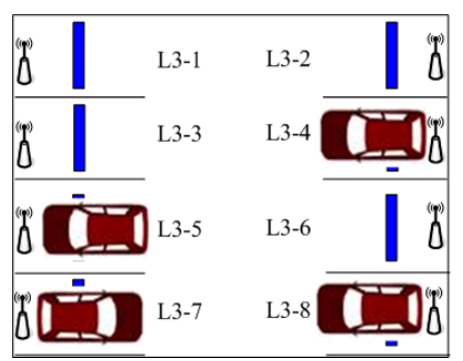

Fig. 6. Parking Spot Detail
Some drivers may consciously or unconsciously misfill the assigned slots. Our assumption in the misparking issue is that the majority of drivers will not mispark. This assumption is reasonable. The majority of people are right people in moral. Moreover, the misparking drivers will be charged a fine. This will help reduce the misparking ratio. Therefore, a small amount of misparking will not hurt the system because the system has fault tolerance. The event whether a slot is filled or not will be dynamically detected and reported to the computer center. Therefore the capacity of vacant slots can be up to date. If the customer who reserved the slot which has been taken by someone else arrives at the booth, he will get a new parking slot. If he does not arrive at the booth yet, he will be informed and assigned a new slot. If the customer is inside the park area and his slot is filled by someone else, he can park at any place and will not receive any fine because computer center can tell who breaks the regulation first by checking the timestamp.

\section{SIMULATION RESULTS}

\section{A. Simulation Settings}

In the simulation, we compare two scenarios. In scenario one, drivers randomly select a parking spot. The parking spot is based on first-come-first-serve. The process that drivers arrive at or exit to the parking lot is a Poisson Process. If they move around the parking area and can not find a parking spot, they turn around. If they turn around 3 times, they exit. In scenario two, drivers reserve a parking spot. The process that drivers arrive at or exit to the parking lot is the same Poisson Process. We assume 1\% of drivers will disobey the reservation and take somebody else's parking spot for their own convenience. The remaining $99 \%$ of drivers obey the reservation. We compare the parking utilization and average waiting time for drivers. The parking capacity is 2000 parking spots. The average arrival rate is 0.5 vehicle/min [AK94]. The simulation parameters and values are listed in Table I.

TABLE I

PARAMETERS AND VALUES

\begin{tabular}{|c|c|}
\hline Parameters & Values \\
\hline Exit booth service rate & 5 vehicles/min \\
Entry booth service rate & 20 vehicles/min \\
Number of exit booths & 5 \\
Number of entry booths & 1 \\
Average arrival rate & 0.5 vehicle/min \\
Average exit rate & 0.5 vehicle/min \\
Average parking time & 1.5 hour \\
\hline
\end{tabular}

First, we evaluated the utilization of parking spots. We varied the capacity of parking lot from 100 spots to 2800 spots. For each day, we simulated 8 hours because we want to simulate daytime parking. An average parking utilization rate is obtained by calculating 7 days' average parking utilization rate. As we expected, the conventional parking approach has less parking spot utilization than our proposed SmartParking, as shown in Figure 7. 


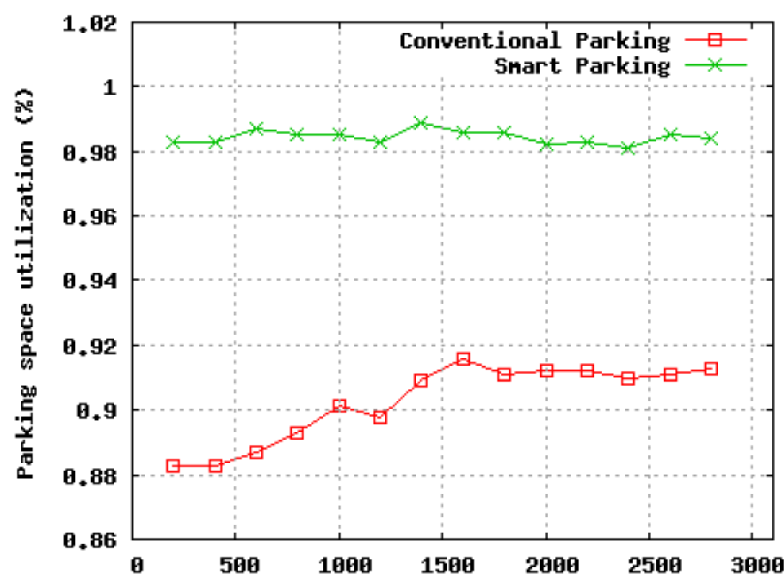

Fig. 7. Parking Utilization

We are interested in how much time a driver uses to search for an empty parking spot inside a parking area. Again we varied the capacity of parking spaces from 100 spots to 2800 spots. We assume $1 \%$ drivers will mispark their cars. And we calculate 7 days' average search time. The search time starts from entry time to the time when an empty parking spot is found. Our proposed SmartParking almost keeps the same amount of time, as we expected, shown in Figure 8. But the conventional parking approach takes more search time as the parking capacity increases.

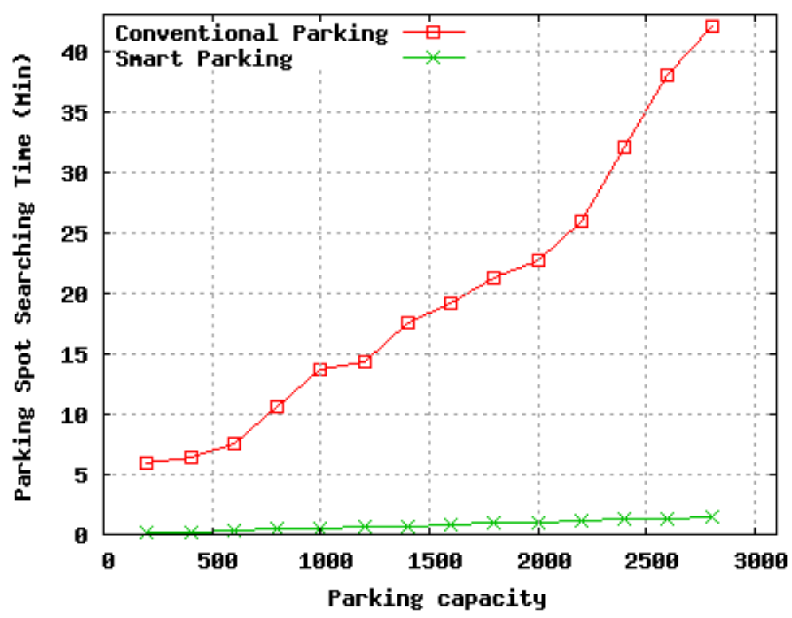

Fig. 8. Parking Spot Searching Time

\section{CONCLUSIONS AND FUTURE WORK}

This paper has proposed a smart parking system. By using the concept and architecture of NOTICE, SmartParking is a intelligent parking service application as well as a novel security/privacy aware infrastructure. First, vehicles on the road can view and reserve a parking spot. The parking process can be an efficient and non-stop service. Second, privacy of the drivers is considered and protected by using the NOTICE infrastructure. Finally, security of the information is protected by using the belt infrastructure and encryption/decryption approach. Simulation results prove the proposed system results in high parking space utilization and fast parking spot finding time.

\section{REFERENCES}

$\left[\mathrm{ABD}^{+} 06\right]$ A. Aijaz, B. Bochow, F. Dötzer, A. Festag, M. Gerlach, R. Kroh, and T. Leinmüller. Attacks on inter-vehicle communication systems - an analysis. In Proceedings of Workshop on Intelligent Transportation (WIT 2006), 2006.

[AK94] Y. Asakura and M. Kashiwadani. Effects of parking availability information on system performance:a simulation model approach. In Proceedings of Vehicle Navigation and Information Systems Conference, pages 251-254, Dearborn, MI, Aug 1994.

[AOW08] M. Abuelela, S. Olariu, and M. C. Weigle. NOTICE: An architecture for notification of traffic incidents. In Proceedings of the IEEE Vehicular Technology Conference - Spring, pages 3001-3005, Singapore, May 2008.

$\left[\mathrm{BOO}^{+} 06\right]$ J. P. Benson, T. O’Donovan, P. O'Sullivan, U. Roedig, and C. Sreenan. Car-park management using wireless sensor networks. In Proceedings of 31st IEEE Conf. Local Computer Networks, pages 588-595, Tampa, 2006.

[CGM06] M. Caliskan, D. Graupner, and M. Mauve. Decentralized discovery of free parking places. In VANET '06: Proceedings of the 3rd international workshop on Vehicular ad hoc networks, pages 30-39, New York, NY, USA, 2006.

[FMO04] S. Funck, N. Mohler, and W. Oertel. Determining car-park occupancy from single images. In Proceedings of International Symposium on Intelligent Vehicles (IVS04), pages 325-328, Parma, Italy, 2004.

[HvL04] J. P. Hubaux, S. Čapkun, and J. Luo. The security and privacy of smart vehicles. IEEE Security and Privacy, 2(3):49-55, 2004.

[MK00] L. E. Y. Mimbela and L. A. Klein. A summary of vehicle detection and surveillance technologies used in intelligent transportation systems, Nov 2000.

[NYC06] NYCDCP. North America CBD Parking Rate Survey Highlights, New York City Department of City Planning. In North America CBD Parking Rate Survey Highlights, August/September 2006.

[PTSP07] R. Panayappan, J. M. Trivedi, A. Studer, and A. Perrig. Vanetbased approach for parking space availability. In VANET '07: Proceedings of the fourth ACM international workshop on Vehicular ad hoc networks, pages 75-76, New York, NY, USA, 2007.

[TYI04] H. Takizawa, K. Yamada, and T. Ito. Vehicles detection using sensor fusion. In Proceedings of International Symposium on Intelligent Vehicles (IVSO4), pages 238-243, Parma, Italy, 2004.

[TZC06] V. Tang, Y. Zheng, and J. Cao. An intelligent car park management system based on wireless sensor networks. In Proceedings Int. Sym. Pervasive Computing and Applications, pages 65-70, Urumqi, 2006.

$\left[\mathrm{WHG}^{+}\right.$06] J. Wolff, T. Heuer, H. Gao, M. Weinmann, S. Voit, and U. Hartmann. Parking monitor system based on magnetic field sensors. In Proceedings IEEE Conf. Intelligent Transportation Systems, pages 1275-1279, Toronto, CA, 2006.

[WO07] M. C. Weigle and S. Olariu. Intelligent highway infrastructure for planned evacuations. In Proceedings of the First International Workshop on Research Challenges in Next Generation Networks for First Responders and Critical Infrastructures (NetCri), pages 594-599, New Orleans, LA, Apr 2007.

[YOW08] G. Yan, S. Olariu, and M. C. Weigle. Providing VANET security through active position detection. Computer Communications: Special Issue on Mobility Protocols for ITS/VANET, 31(12):2883C2897, 2008.

[ZZL07] Z. F. Zhu, Y. Zhao, and H. Q. Lu. Sequential architecture for efficient car detection. In Proceedings of IEEE Computer Society Conference on Computer Vision and Pattern Recognition (CVPR 2007), pages 1-8, Minneapolis, 2007. 